\title{
Estudio retrospectivo: características epidemiológicas de los pacientes con síndrome de apnea e hipoapnea obstructiva del sueño y roncopatía intervenidos por faringoplastia, Hospital Británico de Buenos Aires, 2011-2016
}

\section{Retrospective Study: Epidemiologic characteristics in patients with obstructive sleep apnea and hypopnea syndrome and snoring who underwent pharyngoplasty, Hospital Británico de Buenos Aires: 2011-2016 / Estudo retrospectivo: características epidemiológicas dos pacientes com síndrome de apneia e hipoapneia obstrutiva do sono e roncopatia intervindos por faringoplastia, Hospital Britânico de Buenos Aires, 2011-2016}

Lucía Tapia ${ }^{1}$, María Andrea Ricardo², Pablo Rubén Fernández ${ }^{3}$, Alberto Eugenio Rabino ${ }^{4}$

\section{RESUMEN}

Objetivo: el síndrome de apnea e hipoapnea obstructiva del sueño (SAHOS) y las roncopatías contribuyen al desarrollo de comorbilidades. La primera línea de tratamiento del SAHOS es el CPAP. En pacientes sin adhesión al tratamiento, se propone la opción quirúrgica. Se han descrito múltiples intervenciones adaptadas a cada paciente de acuerdo al grado de obstrucción. Este estudio describe las características epidemiológicas de los pacientes intervenidos por faringoplastias en el periodo comprendido entre 2011 y 2016 en el Hospital Británico de Buenos Aires.

Metodología: estudio retrospectivo de pacientes intervenidos por faringoplastias. Se incluyeron pacientes mayores de 18 con diagnóstico de SAHOS por polisomnografía y/o roncopatía. Se excluyeron pacientes menores de 18 años y con obesidad mórbida. Resultados: se incluyeron 51 pacientes con una edad media de $44.8 \pm 1.7$ años, de los cuales el $84.3 \%$ fueron hombres. $35.3 \%(n=18)$ de los pacientes presentaron alguna comorbilidad, siendo la más frecuente hipertensión arterial. $85 \%(n=43)$ de los pacientes presentaban SAHOS, siendo un $29,4 \%$ severo y el $35,3 \%$ de grado moderado. $15 \%$ de los pacientes que optaron por el tratamiento quirúrgico presentaban roncopatía.

Conclusiones: en este estudio se encontró que los pacientes fueron en su mayoría hombres con una edad media de 44.8 \pm 1.7 , sintomáticos (Epworth > 9), con obesidad (IMC $28.9 \pm 0.4)$ y con baja adherencia al CPAP. La comorbilidad más frecuente fue hipertensión arterial. El grado de SAHOS prevalente fue el moderado (35,5\%). 15\% de los pacientes que optaron por el tratamiento quirúrgico presentaban roncopatía.

Palabras clave: síndromes de la apnea del sueño; ronquido; procedimientos quirúrgicos otorrinolaringológicos.

\section{ABSTRACT}

Objective: Obstructive sleep apnea and hypopnea syndrome (OSAHS) and snoring contribute to the development of comorbidities. The first line of treatment in OSAHS is CPAP. In patients without treatment adherence, the surgical option is proposed. Multiple interventions adapted to each patient have been described according to degree of obstruction. This study describes the epidemiological characteristics of patients who
Fecha de recibido: 31 de enero de 2018

Fecha de aprobación: 17 de mayo de 2018

Forma de citar este artículo: Tapia L, Ricardo MA, Fernández PR, Rabino AE. studio retrospectivo: características epidemiológicas de los pacientes con síndrome de apnea e hipoapnea obstructiva del sueño y roncopatía intervenidos por faringoplastia, Hospital Británico de Buenos Aires, 2011-2016. Med UPB. 2019;38(1):11-16. DOI:10.18566/medupb.v38n1.a02

1. Residente III año otorrinolaringología, Hospital Británico de Buenos Aires. Buenos Aires, Argentina.

2. Médica, Otorrinolaringóloga. Jefe de servicio otorrinolaringología, Hospital Británico de Buenos Aires. Buenos Aires, Argentina.

3. Médico, Otorrinolaringólogo. Médico de planta, Hospital Británico de Buenos Aires. Buenos Aires, Argentina.

4. Médico, Otorrinolaringólogo. Médico de planta Hospital Británico de Buenos Aires. Buenos Aires, Argentina.

Dirección de correspondencia: Lucía Tapia. Correo electrónico: lutapia88@gmail.com 
underwent pharyngoplasty between 2011 and 2016 at the Hospital Británico de Buenos Aires.

Methodology: Retrospective study of patients who underwent pharyngoplasty. Patients over 18 with OSAHS diagnosed by polysomnography and/or chronic snoring were included. We excluded patients under 18 as well as those with morbid obesity.

Results: 51 patients were included with an average age of $44.8 \pm 1.7$, of whom $84.3 \%$ were men. $35.3 \%(n=18)$ of the patients presented some comorbidity, where arterial hypertension was the most frequent. $85 \%(n=43)$ of the patients had OSAHS, of which $29.4 \%$ involved a severe degree and $35.3 \%$ a moderate one. $15 \%$ of patients who underwent surgical treatment presented snoring.

Conclusions: In this study, it was found that the patients were mostly men with a mean age of $44.8 \pm 1.7$; they were also symptomatic (Epworth $>9$ ), suffered from obesity (BMI $28.9 \pm 0.4$ ), and presented poor adherence to CPAP. The most frequent comorbidity was arterial hypertension. The prevalent degree of OSAHS was moderate (35.5\%). $15 \%$ of patients who underwent surgical treatment suffered from chronic snoring.

Key words: sleep apnea syndromes; snoring; otorhinolaryngologic surgical procedures.

\section{RESUMO}

Objetivo: o síndrome de apneia e hipoapneia obstrutiva do sono (SAHOS) e as roncopatias contribuem ao desenvolvimento de comorbilidades. A primeira linha de tratamento do SAHOS é o CPAP. Em pacientes sem adesão ao tratamento, se propõe a opção cirúrgica. Se há descrito múltiplas intervenções adaptadas a cada paciente de acordo ao grau de obstrução. Este estudo descreve as características epidemiológicas dos pacientes intervindos por faringoplastias no período compreendido entre 2011 e 2016 no Hospital Britânico de Buenos Aires.

Metodologia: estudo retrospectivo de pacientes intervindos por faringoplastias. Se incluíram pacientes maiores de 18 com diagnóstico de SAHOS por polisomnografia e/ ou roncopatia. Se excluíram pacientes menores de 18 anos e com obesidade mórbida. Resultados: se incluíram 51 pacientes com uma idade média de $44.8 \pm 1.7$ anos, dos quais 84.3\% foram homens. $35.3 \%(n=18)$ dos pacientes apresentaram alguma comorbilidad, sendo a mais frequente hipertensão arterial. $85 \%(n=43)$ dos pacientes apresentavam SAHOS, sendo um $29,4 \%$ severo e $35,3 \%$ de grau moderado. $15 \%$ dos pacientes que optaram pelo tratamento cirúrgico apresentavam roncopatia.

Conclusões: neste estudo se encontrou que os pacientes foram na sua maioria homens com uma idade média de 44.8 \pm 1.7 , sintomáticos (Epworth > 9), com obesidade (IMC $28.9 \pm 0.4)$ e com baixa aderência ao CPAP. A comorbilidade mais frequente foi hipertensão arterial. O grau de SAHOS prevalente foi o moderado (35,5\%). $15 \%$ dos pacientes que optaram pelo tratamento cirúrgico apresentavam roncopatia.

Palavras chave: síndromes da apneia do sono; ronquido; procedimentos cirúrgicos otorrinolaringológicos.

\section{INTRODUCCIÓN}

Los trastornos respiratorios obstructivos del sueño (TROS) incluyen roncopatías y síndrome de apnea e hipoapnea obstructiva del sueño (SAHOS). Estos son obstrucciones de las vías aéreas superiores (VAS), que requieren la intervención por parte de equipos multidisciplinarios ${ }^{1}$. Se ha demostrado que los pacientes con TROS presentan comorbilidades, por lo que requieren un adecuado diagnóstico y tratamiento temprano ${ }^{2}$.

El ronquido es uno de los signos clínicos más comunes del SAHOS. Se estima que aproximadamente un $45 \%$ de hombres y un 30\% de mujeres mayores de 65 años presentan roncopatía ${ }^{2}$. El SAHOS es considerado un desorden común en la población adulta, cuyo reconocimiento se encuentra en ascenso, con una prevalencia de $3 \%$ a $7 \%$ en hombres y de $2 \%$ a $5 \%$ en 
mujeres. El patrón de referencia para el diagnóstico del SAHOS es la polisomnografía (PSG), la cual permite identificar y clasificar las apneas e hipoapneas presentes durante el sueño ${ }^{3}$.

La elección del tratamiento debe ser específica e individualizada para cada paciente, teniendo en cuenta factores anatómicos, índices de severidad de enfermedad, comorbilidades asociadas, adherencia al tratamiento y posibilidad de seguimiento. Actualmente se considera tratamiento de primera línea el uso del CPAP (continuous positive airway pressure) en pacientes con SAHOS, aunque la adherencia al mismo resulta un problema evidente ( $46 \%$ a $83 \%$ no logran adaptarse), por lo que se elige la opción quirúrgica en estos pacientes ${ }^{4,5}$.

Se han descrito múltiples intervenciones, como amigdalotomías, faringoplastias laterales, uvulopalatofaringoplastias, cirugías de avance mandibular y cirugías multinivel que, de acuerdo a diversos criterios, han cambiado acorde al tipo de paciente y al grado de obstrucción del mismo ${ }^{6}$. La uvulopalatofaringoplastia, descrita por Fujita $e t a l^{7}$., en el año 1981, ha sido cuestionada y se han recomendado diversas modificaciones en las últimas décadas. Cahali et al., describió la faringoplastia lateral, y luego Pang y Tucker Woodson en 2007 describieron la faringoplastia expansiva (Expansion Sphinteric Pharingoplasty), entre otros ${ }^{8}$. Entre los procedimientos más utilizados actualmente se encuentran la amigdalectomía, uvulopalatofaringoplastia, esfinterofaringoplastia expansiva, ablación con radiofrecuencia de base de lengua, faringoplastia lateral, colgajo uvulopalatino y cirugía de avance mandibular ${ }^{9}, 10$.

Existe una gran heterogeneidad entre los estudios publicados en América del Norte, América del Sur, Europa y Oceanía que describen o comparan las diferentes técnicas quirúrgicas a nivel faríngeo para el tratamiento del SAHOS y las roncopatías. Se trata de muestras pequeñas, con diversos criterios de inclusión e indicación terapéutica de los pacientes y cortos periodos de seguimiento. Los criterios de inclusión y las indicaciones principales para el tratamiento quirúrgico en estos pacientes incluyen la no adherencia al CPAP, edad entre 18-70 años, IMC $<40$, SAHOS moderado a severo, escala de Epworth $>9$ y la voluntad del paciente ${ }^{5,6,9,11,12}$.

Este estudio se propone describir las características epidemiológicas de los pacientes intervenidos por faringoplastia.

\section{METODOLOGÍA}

Se realizó un estudio retrospectivo observacional de pacientes intervenidos por faringoplastias en el servicio de otorrinolaringología del Hospital Británico de Buenos Aires -hospital de cuarto nivel- entre junio de 2011 y diciembre de 2016. Este estudio fue aprobado por el
Comité de Revisión Institucional del Hospital Británico de Buenos Aires en base a los criterios de Helsinki.

Se incluyeron pacientes intervenidos por faringoplastia, que consultaron por roncopatía o SAHOS en el servicio de otorrinolaringología en el período establecido. Entre los procedimientos que se realizaron se incluyen faringoplastia lateral, colgajo uvulopalatino y esfinterofaringoplastia expansiva. Se excluyeron aquellos pacientes en quienes no se optó por el tratamiento quirúrgico, aquellos menores de 18 años y pacientes con obesidad grado II o mórbida (IMC $\geq 35$ ).

Se registraron variables tales como edad, género, motivo de consulta, índice de masa corporal (IMC), escala de Epworth (ESS), índice de apneas e hipoapneas (IAH), diagnóstico definitivo y comorbilidades asociadas a través de la revisión de historias clínicas y protocolos quirúrgicos de una muestra significativa de pacientes.

El análisis estadístico de las variables se resumió en tablas y gráficos, con medidas de frecuencia absoluta y relativa, así como de tendencia central y de variación. Se realizó utilizando el software PSPP, versión 10.1. Se aplicó una estadística descriptiva simple para caracterizar la muestra y se utilizó la t de Student o la prueba Chicuadrado para significancia estadística según el valor de $\mathrm{p}<0.05$.

\section{RESULTADOS}

Se incluyeron en este estudio 51 pacientes de los cuales el $84.3 \%$ eran de sexo masculino cuyas características se detallan en la tabla 1 . La edad promedio de los pacientes fue de $47.7 \pm 4.5$ para las mujeres y de $44.3 \pm 1.9$ años para los hombres, la diferencia entre géneros no es significativa $(p=0.45)$ y en un rango entre 26 años y 68 años. Se encontró una diferencia significativa, con mayor IMC en los hombres comparados con las mujeres (Tabla 1).

Un 39.2\% de los pacientes operados eran obesos (IMC $>30$ ) y un $50.9 \%$ presentaban sobrepeso.

Los hallazgos de este estudio mostraron que el $60.8 \%$ de los pacientes $(\mathrm{n}=31)$ acudieron a la consulta por roncopatía. El 39.2\% de los casos $(n=20)$ consultaron por roncopatía y con diagnóstico confirmado de SAHOS, presentando baja adherencia al tratamiento instaurado, ya sea por fallas en la utilización del CPAP o por baja tolerancia al mismo.

En la anamnesis y en el examen físico se tomaron datos como ESS, IMC, grado de amígdalas palatinas y Friedman Tongue Position. Dentro de los valores obtenidos en la ESS, el 37\% de las mujeres presentó valores mayores a 9 , mientras que en los hombres un $72 \%(\mathrm{p}=0.09)$.

E1 $72.5 \%$ de los pacientes intervenidos $(\mathrm{n}=37)$ no presentaban comorbilidades asociadas, mientras que un $27.5 \%$ presentó al menos una comorbilidad $(\mathrm{n}=14)$. Solo 14 pacientes presentaron comorbilidades, cuatro mujeres 
ARTÍCULO ORIGINAL - ORIGINAL ARTICLE - ARTIGO ORIGINAL | Estudio retrospectivo: características epidemiológicas de los pacientes con síndrome de apnea e hipoapnea obstructiva del sueño y roncopatía intervenidos por faringoplastia, Hospital Británico de Buenos Aires, $2011-2016$

Tabla 1. Características clínico-demográficas de los pacientes intervenidos por faringoplastias.

\begin{tabular}{lccc}
\hline & Mujeres & Hombres & Valor de p \\
\hline $\mathrm{n}$ & 8 & 43 & \\
Edad (años) & $47.7 \pm 4.5$ & $44.3 \pm 1.9$ & 0.450 \\
Rango etario & $30-63$ & $26-68$ & \\
$\mathrm{IMC}(\mathrm{kg} / \mathrm{m} 2)$ & $25.76 \pm 0.9$ & $29.43 \pm 0.5$ & 0.003 \\
$\mathrm{ESS}^{*}$ & $10.25 \pm 2.3$ & $11.74 \pm 0.84$ & 0.330 \\
ESS $>$ 9 (\%) & 37 & 72 & 0.090 \\
$\mathrm{~N}^{\circ}$ pacientes con comorbilidades & 4 & 10 & 0.190 \\
\hline
\end{tabular}

y diez hombres. No se observaron diferencias respecto al porcentaje de comorbilidades por género, siendo la HTA la comorbilidad más frecuente. Se observó que en mujeres predominaban las alteraciones tiroideas como el hipotiroidismo, mientras que en hombres se evidenciaron otras comorbilidades como arritmia, epilepsia y colon irritable (Figura 1).

Los resultados de las PSG preoperatorias, permitieron analizar la severidad de los pacientes por los valores del índice de apneas/hipoapneas (IAH) y por el porcentaje de tiempo que los pacientes desaturan por debajo del $90 \%$ (T90). Se observó que el valor de IAH de los pacientes era muy variable, en un rango de 0.5 a 80 , con una media de 27,4 con un T90 que se mantuvo en un rango de $62 \%$ a $92 \%$, con una media de $81.4 \%$.

En base al IAH, se observó que la severidad del SAHOS más frecuentemente encontrado en la muestra fue de moderado-severo para ambos sexos. El 64.7\% de los pacientes estudiados $(\mathrm{n}=33)$ presentaron un SAHOS de grado moderado-severo, mientras que un $15.7 \%$ de los pacientes obtuvieron una PSG normal con un IAH menor a 5 (Figura 2).

\section{DISCUSIÓN}

La medicina del sueño, considerada una nueva ciencia, estudia las roncopatías y el SAHOS y ha tenido un crecimiento exponencial en los últimos años. El trastorno del sueño más común en la población general actualmente es el SAHOS ${ }^{2}$.

En trabajos latinoamericanos como el de Almeida Mendes $^{2}$ en Brasil, se presentó una población en la que el principal motivo de consulta fue el ronquido (un $90 \%$ de los casos). El autor establece que un $45 \%$ de los hombres

Figura 1. Comorbilidades en los pacientes intervenidos por faringoplastia $(n=14)$

A.

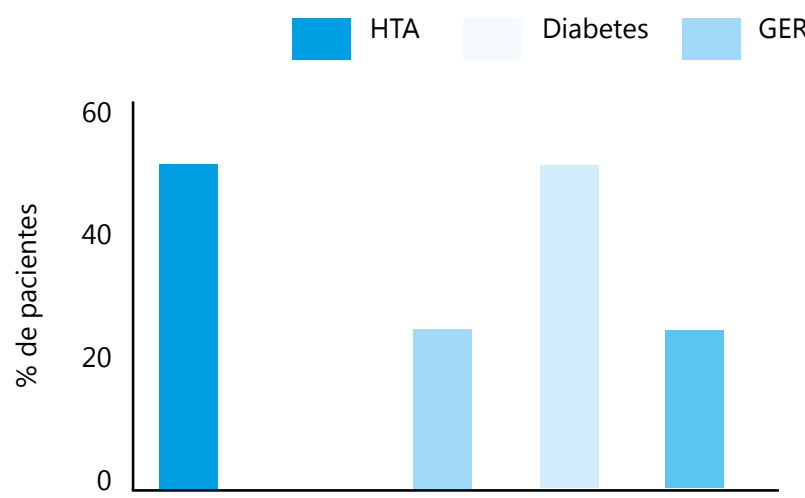

B.

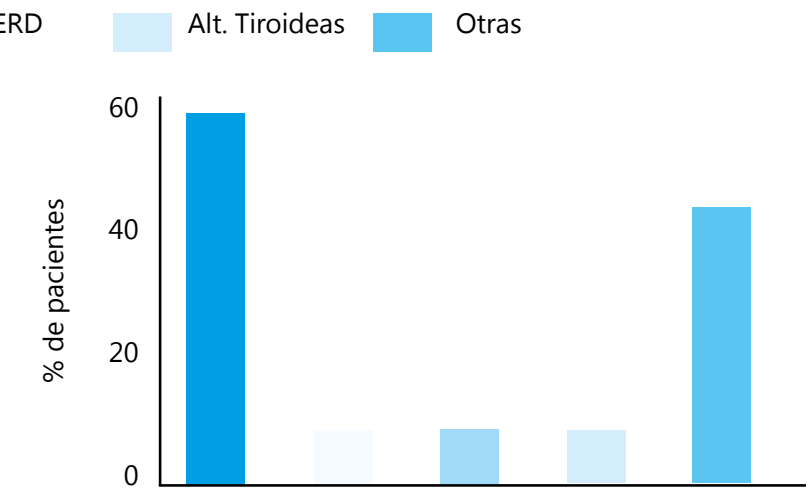

A) mujeres, B) hombres. HTA hipertensión arterial. GERD enfermedad por reflujo gastroesofágico. ALT TIROIDEAS alteraciones tiroideas. 
Figura 2. Descripción de la gravedad del SAHOS en los pacientes intervenidos, diferenciada por género

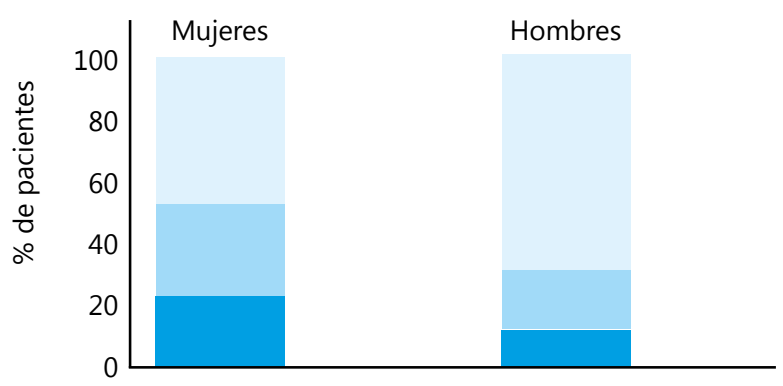

Norma

Leves

Moderado/Severo

y un 30\% de las mujeres mayores a 65 años presentan roncopatía. Por lo tanto, considera a la roncopatía como una condición para evaluar e investigar otros TROS ${ }^{2}$.

La Asociación Americana de la Medicina del Sueño (AASM) considera al CPAP el tratamiento de primera línea y el gold estándar en el tratamiento del SAHOS ${ }^{1}$ Mackay $^{4}$, Robinson ${ }^{11}$ y Rotemberg ${ }^{12}$ coinciden con esta declaración y estudiaron la respuesta y la efectividad de este tratamiento. Se considera adherencia al CPAP un mínimo de uso de cuatro horas por noche, por cinco noches a la semana (70\%), durante 30 días consecutivos o tres meses ${ }^{11}$. Entre un $46 \%$ y un $83 \%$ de los pacientes con SAHOS fracasan en la adherencia al CPAP. Hasta un $50 \%$ lo abandonan en una semana, y entre $12 \%$ y $25 \%$ en los primeros tres años. Es en dichos pacientes en los que se indica el tratamiento quirúrgico ${ }^{5,11,12}$. En nuestro medio, el tratamiento con CPAP y el seguimiento y adaptación al mismo depende principalmente del servicio de neumonología, encargado de derivar aquellos pacientes en quienes fracasa el tratamiento al otorrinolaringólogo para evaluar opciones terapéuticas de segunda línea.

La cirugía como tratamiento del SAHOS ha crecido considerablemente en los últimos años, con numerosas modificaciones de las distintas técnicas quirúrgicas disponibles, siendo la más comúnmente utilizada la faringoplastia y sus variantes, con o sin amigdalectomía 4 .

Nuestros resultados, en concordancia con la literatura, mostraron que los pacientes intervenidos tuvieron predominancia masculina. Dentro de los principales factores predisponentes y comorbilidades asociadas descritas en la bibliografía, se encuentra la obesidad, el tabaquismo, la diabetes, la depresión, el asma y las alteraciones cardiovasculares ${ }^{4,11-15}$. El grado de obesidad y el grado de severidad del SAHOS, constituyen dos variables que pueden relacionarse ${ }^{15}$. Randerath ${ }^{9}$, en las guías alemanas, estableció como límite para el tratamiento quirúrgico un
IMC mayor a 40. En cambio, Rotemberg ${ }^{12}$ en Canadá, estableció un IMC mayor a 35 como límite para realizar un procedimiento quirúrgico. Se excluyen de los candidatos a tratamientos quirúrgicos aquellos pacientes con obesidad grado II u obesidad mórbida.

Aurora $^{6}$, en su actualización sobre los parámetros prácticos a la hora de modificar quirúrgicamente la anatomía de los pacientes con SAHOS, estableció la importancia de determinar la presencia y severidad de la patología antes de la cirugía. Como establece la AASM, se calificaron los grados evolutivos determinados por el IAH en normal (<5), leve ( 5 a 15$)$, moderado ( 16 a 30 ) y severo $(>30)^{1}$. Los autores indicaron el tratamiento quirúrgico en aquellos pacientes con un IAH mayor a 15. Esta indicación se mantiene en los pacientes intervenidos en nuestro estudio, en el que aproximadamente el 65\% de los pacientes variaban entre un SAHOS moderado y un SAHOS severo.

Otras indicaciones tomadas en consideración para el tratamiento quirúrgico corresponden a una escala de Epworth $>9$, las características anatómicas de la vía aérea superior de cada paciente y, principalmente, la calidad de vida y el deseo de los pacientes por optar por esta línea de tratamiento.

Es importante recalcar que este es un estudio piloto y constituye un primer paso para el diseño de un estudio de cohorte donde se analizarían los resultados del tratamiento quirúrgico en nuestro medio.

En conclusión, en este estudio, se observó mayor indicación quirúrgica de faringoplastias en pacientes de sexo masculino en la cuarta década de la vida sin comorbilidades asociadas. El principal motivo de consulta en los pacientes intervenidos fue la roncopatía. La comorbilidad más frecuentemente encontrada fue la hipertensión arterial en ambos sexos. El índice de gravedad del SAHOS más frecuente, según la estrategia de medición usada, fue el moderado-severo. Finalmente, la media de la muestra presentaba una escala de Epworth $>9$ y un IMC $>25$, indicando sobrepeso. Cabe destacar que ningún paciente intervenido presentó un IMC >40, siendo congruente con lo establecido en la bibliografía.

\section{DECLARACIÓN DE CONFLICTO DE INTERESES}

Los autores declaran no tener ningún conflicto de interés. Este estudio fue aprobado por el Comité de Revisión Institucional del Hospital Británico de Buenos Aires. 


\section{REFERENCIAS}

1. American Academy of Sleep Medicine. The International Classification of Sleep Disorders, Revised. Diagnostic and Coding Manual. 2001.

2. Almeida F, Monteiro S, Duarte B, Parsekian A. Epidemiologic profile of patients with Snoring and Obstructive Sleep Apnea in a University Hospital. Int Arch Otorhinolaryngol 2014; 18:142-145.

3. Punjabi N. The Epidemiology of Adult Obstructive Sleep Apnea. Proc Am Thorac Soc 2008; 5:136-143.

4. Mackay S, Jefferson N, Marshall N. Beyond Uvulopalatopharyngoplasty for obstructive sleep apnea. Single surgeon case series of contemporary airway reconstruction. Journal of Laryngology and Otology 2013; 127:1184-1189.

5. Weaver T, Grunstein R. Adherence to continuous positive Airway pressure therapy. The challenge to effective treatment. Proceedings of the American Thoracic Society 2008; 5:173-178.

6. Aurora N, Casey K, Kristo D, Auerbach S, Bista S, Chowdhuri S, et al. Practice parameters for the surgical modifications of the upper airway for obstructive sleep apnea in adults. Sleep 2010; 33(10):1408-1413.

7. Fujita S, Cinway W, Zorik F. Surgical Correction of anatomic abnormalities in Obstructive Sleep Apneas Syndrome: Uvulopalatopharyngoplasty. Otolaryngol Head Neck Surg 1981; 89:923-934.

8. Emara T, Hassan M, Mohamad A, Anany A, Ebrahem A. Anterolateral advancement pharyngoplasty: A new technique for treatment of Obstructive Sleep Apnea. Otolaryngol Head Neck Surg 2016; 155(4):702-707.

9. Randerath W, Verbraecken J, Andreas S, Bettega G, Boudewyns A, Hamans E, et al. Non - CPAP therapies in Obstructive Sleep Apnea. ERS Task Force Report. Eur Respir J 2011; 37:1000-1028.

10. Zancanella E, Haddad FM, Oliveira LA, Nakasato A, Duarte B, Soares CF, et al. Guideline. Obstructive sleep apnea and primary snoring: treatment. Braz J Otorhinolaryngol 2014; 80(1):517-528.

11. Robinson S, Chia M, Carney S, Chawla S, Harris P, Esterman A. Upper Airway reconstructive surgery long - term quality of life outcomes compared with CPAP for adults Obstructive Sleep Apnea. Otolaryngol Head Neck Surg 2009; 141:257-263.

12. Rotenberg B, Theriault J, Gottesman S. Redefining the timing of surgery for Obstructive Sleep Apnea in Anatomically Favorable Patients. Laryngoscope 2014; 124:51-59.

13. Epstein L, Kristo D, Strollo P, Friedman N, Malhotra A, Patil S, et al. Clinical Guideline for the evaluation, management and long-term care of Obstructive Sleep Apnea un adults. Journal of Clinical Sleep Medicine 2009; 5(3):263-276.

14. Friedman M, Ibrahim H, Lowenthal S, Ramakrishnam V, Joseph N. Uvulopalatoplasty: A modified technique por selected patients. Laryngoscope 2004; 114:441-449.

15. Pinto JA, Ribeiro D, Cavallini A, Duarte C, Freitas G. Comorbidities Associated with Obstructive Sleep Apnea: A Retrospective Study. Int Arch Otorhinolaryngol 2016; 20:145-150. 